Experimental Research

\title{
The use of postnatal developmental cartilaginous lines in the classification of $\mathrm{C} 2$ fractures
}

\author{
Cengiz Çokluk*, Keramettin Aydın \\ Department of Neurosurgery, Medical Faculty, Ondokuz Mayis University, Samsun, Turkey
}

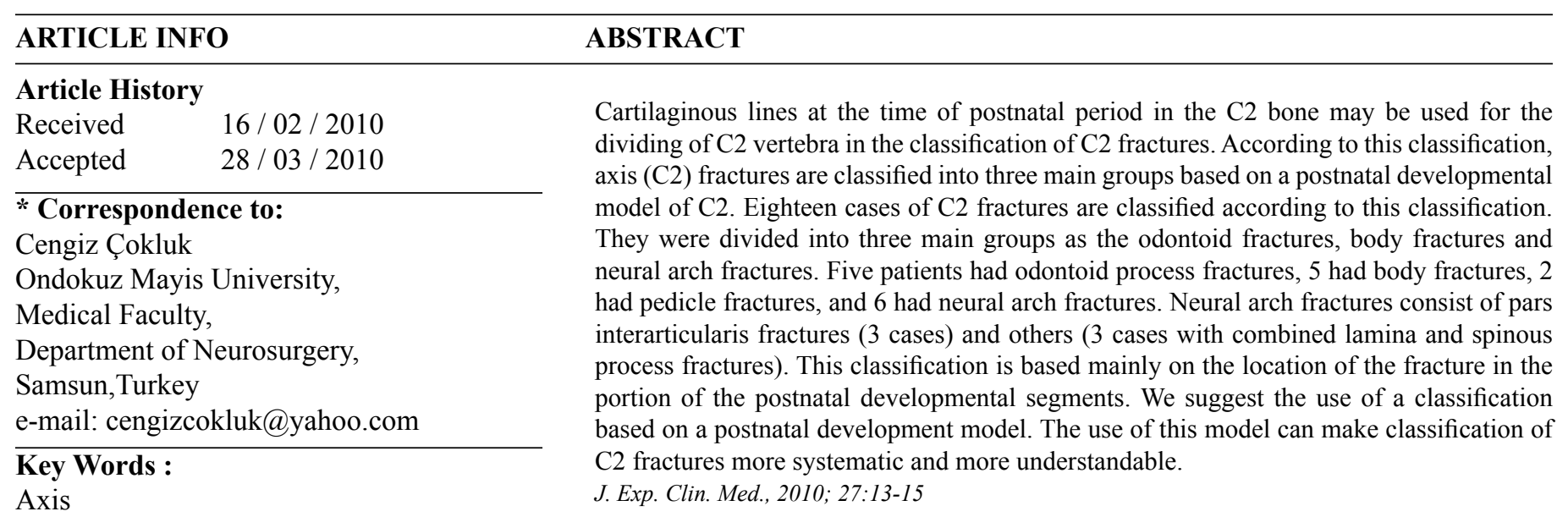

C2 Fractures

Postnatal Development Model

Classification

Cortilaginous

Lines

C) 2010 OMU All rights reserved

\section{Introduction}

In common clinical practice, cervical spine can be divided as upper and lower region. The upper cervical spine consists of the foramen magnum, paired occipital condyles, $\mathrm{C} 1$ and $\mathrm{C} 2$. The upper cervical spine functionally serves as a transitional zone between the rigid calvarium and the lower cervical spine. Different and specific anatomic shape of the structures of upper cervical spine and the ligamentous structures of this zone are responsible for elasticity and flexibility of this area. The largest anatomical structure in this region is the second cervical vertebra also known as axis. The axis functionally is the pivot on which the atlas and hence the head rotate. It morphologically consists of a body, paired pedicles, the lateral masses, the laminae and the bifid spinous process. The odontoid process of $\mathrm{C} 2$ projects vertically upward from the body of $\mathrm{C} 2$ and is also named as dens of axis, a strong toothlike process.

Traumatic cervical spinal column fractures are frequently encountered pathology in the neurosurgical practice. In the other hand the fractures of $\mathrm{C} 2$ are uncommon and had been infrequently reported in the medical literature. Fractures of $\mathrm{C} 2$ account for $17 \%$ of all cervical spine fractures. Fractures of the odontoid and pars interarticularis are well known in clinical practice. These types of fractures were previously described (Anderson and D'Alonzo, 1974; Benzel et al., 1994; Doherty and Heggeness, 1995; Benzel, 1996). The axis may be fractured in every region such as odontoid process, body, pedicle, neural arch, lamina and spinous process. Odontoid fractures are the most common $\mathrm{C} 2$ fractures encountered in clinical practice. Hangman's C2 fractures are other classified fractures of $\mathrm{C} 2$. Nonodontoid and nonhangman's C2 fractures have been identified.

The general classification of $\mathrm{C} 2$ fractures is based on the location of the fractures such as odontoid fractures, body fractures and pedicle fractures. In neurosurgical practice, a simple, systematic classification is needed for description of $\mathrm{C} 2$ fractures. In this clinical study, we propose a new classification method for $\mathrm{C} 2$ fractures based on the postnatal development of $\mathrm{C} 2$.

\section{Materials and Methods}

The fractures were grouped according to the location of the fracture on the postnatal developmental portion of C2. All fractures were divided mainly into three groups as odontoid fractures, body fractures, and neural arch fractures. Segmentation of C2: 
Odontoid Segment: The odontoid process was divided into three parts according to its embryologic and functional properties; the tip, the midportion and the base. The tip of the odontoid is the most superior part of this process. The midportion begins from the end of the tip and extends to the line drawn between the most medial ends of the superior articulating facets. The third part is the base of the odontoid. This portion begins from the inferior border of the midportion and ends at the level of dentocentral synchondrosis (Fig. 1). Body of C2: This is the most inferior segment of $\mathrm{C} 2$. The superior border of this segment is formed by dentocentral synchondrosis. This line is also the inferior border of the odontoid base, as mentioned above (Fig. 1). Neural Arches: Neural arches are the two hemistructures located bilaterally (Fig. 2).

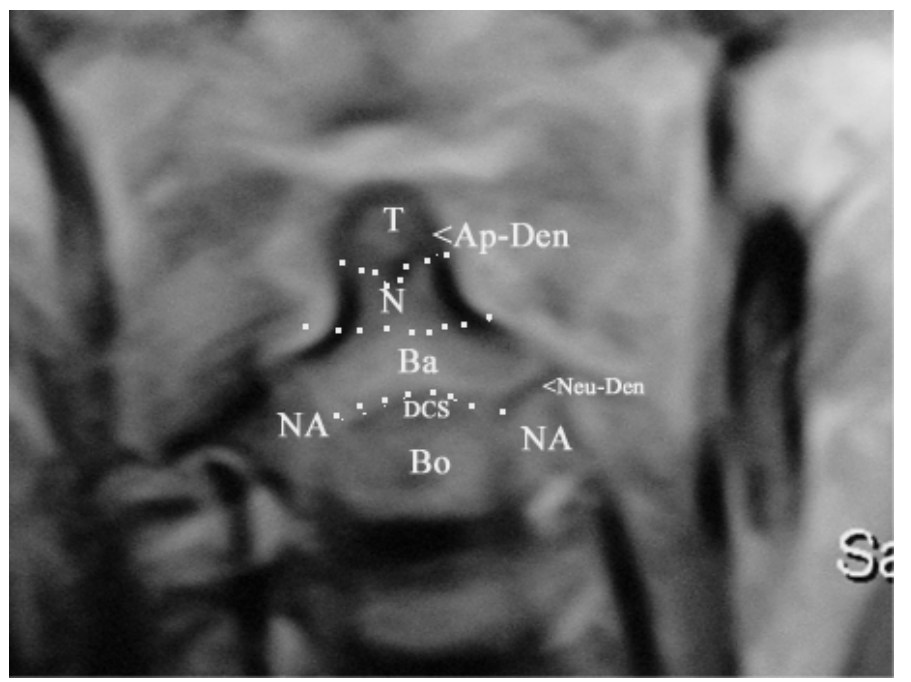

Fig.1. Coronal T1-weighted magnetic resonance imaging of a pediatric case shows the segments of the odontoid process (T: Tip of the odontoid, N: The neck of the odontoid, Ba: The base of the odontoid, Bo: The body of $\mathrm{C} 2$, Ap-Den: Apicodental synchondrosis Neu-Den: Neurodental synchondrosis, dotted areas mark the borders of the segments of the odontoid process).

\section{Results}

The majority of the patients (11/18), (55\%) were involved in traffic accidents; the remaining injuries were bicycle accidents (1/18), (6\%), falls $(5 / 18),(27 \%)$ and diving accidents $(1 / 18),(6 \%)$. All of the patients suffered from neck pain. All of the patients had traumatic scalp abrasions. Conventional radiologic examination of $\mathrm{C} 2$ showed $\mathrm{C} 2$ C3 angulations with odontoid process fracture in one case $(6 \%)$, C4-C5 anterior dislocation with hangman fractures in one case $(6 \%)$. Type II odontoid fractures and pars interarticularis fractures were easily detected by direct X-ray. CT scan is helpful in the evaluation of $\mathrm{C} 2$ and the skull. Skull fractures associated with $\mathrm{C} 2$ fracture were present in 5 cases $(28 \%)$. CT with bone window was especially helpful in the determination of pedicle and body fractures. MRI is a helpful diagnostic method in the evaluation of nerves and the perineural ligamentous structures. Five cases $(28 \%)$ had odontoid fractures. The fracture was located in two cases $(40 \%)$ on the base of the odontoid process (Type III fracture). The fracture line were on the midportion of the odontoid in remaining three cases $(60 \%)$ (Type II fracture). This population did not include any apical odontoid fracture. Five cases $(28 \%)$ had body fractures. Two cases $(12 \%)$ had pedicle fractures. Three cases $(16 \%)$ had pars interarticularis fractures. The remaining three cases $(16 \%)$ had other types of neural arches fractures (laminae, spinous processes etc.).

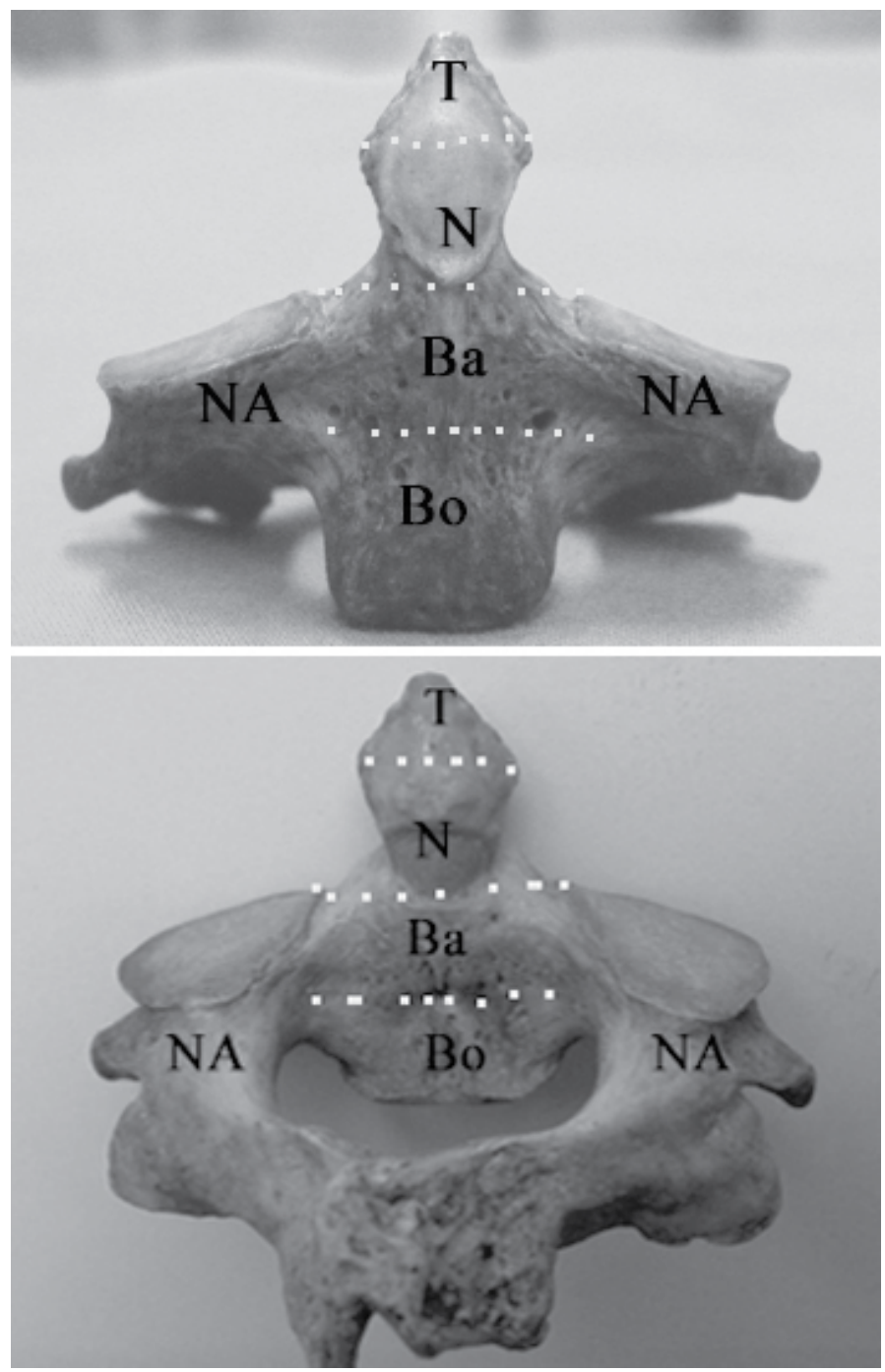

Fig.2. A. This figure taken from a cadaver C2 shows the segments of the odontoid process in antero-posterior view (T: Tip of the odontoid process, $\mathrm{N}$ : Neck of the odontoid process, Ba: Base of the odontoid process, Bo: Body of C2, NA: Neural arc, dotted areas mark the borders of the segments of the odontoid process)

B. This figure taken from a cadaver $\mathrm{C} 2$ shows the segments of the odontoid process in posterior-anterior view (T: Tip of the odontoid process, $\mathrm{N}$ : Neck of the odontoid process, Ba: Base of the odontoid process, Bo: Body of C2, NA: Neural arc, dotted areas mark the borders of the segments of the odontoid process)

\section{Discussion}

We propose a classification based on postnatal developmental segments of the axis. This structure includes three main parts at the birth; the odontoid segment, the body and the neural arches segments. We used these segments as the main determinants for the basic classification of $\mathrm{C} 2$ fractures. This classification includes three types of the fractures; odontoid, body, and neural arch fractures. Neural arch fractures cover pedicle fracture, superior and inferior articulating facet fractures, pars interarticularis fractures, laminae and spinous process fractures.

During the developmental process, the segments of $\mathrm{C} 2$ are separated from each other by synchondroses 
articulations (Doherty et al., 1993). The odontoid proces is joined to the body and neural arches via the dentocental and neurocental syncondrosis respectively (Doherty et al., 1993). The neurocentral synchondrosis connects the neural arches to the base of the odontoid and the body of $\mathrm{C} 2$. This portion is the pedicle of $\mathrm{C} 2$ and gradually ossifies into a bony structure.

The odontoid segment develops from two portions; the apical and body portions. According to this classification, odontoid fractures should be divided into two groups as apical fractures and body fractures. But, the odontoid process is not only an anatomical structure but also a very important and specific functional structure (Doherty et al., 1993). Because of this, the classification of odontoid process should be made according to both of embryological and functional properties.

Anderson and D'Alanzo have classified the odontoid fractures into three groups (Anderson and D'Alonzo, 1974). This classification is the most commonly used in neurosurgical practice. For these reasons, we divided odontoid fractures into three groups. The first group is apical portion fractures, the second group is midportion fractures and the third group includes base fractures. The exact mechanism of odontoid fractures is unknown. However, it consists of a complex mechanism of flexion, extension, and rotation (Doherty et al., 1993).

Benzel classified axis body fractures according to orientation of the fracture line (Benzel et al., 1994). Type I was described as a coronally oriented vertical fracture, Type II was a sagittally oriented vertical fracture and Type III a horizontal rostral fracture. In this classification, the horizontal fracture is rostrally located in the $\mathrm{C} 2$ body and is the same injury as the previously described Type III odontoid process fracture of Anderson and D'Alanzo. This classification is complex and difficult to use in clinical practice. The present patient population includes 4 vertebral body fractures. The type of the fractures is a teardrop avulsion fracture of the anterior caudal aspect of the body of $\mathrm{C} 2$. The mechanism of these types of injuries is hyperextension with axial loading (Benzel et al., 1994).

The nomenclature and classification of axis fractures are important because of the frequency of occurrence and clinical importance. We propose a new classification method based on the postnatal developmental model of axis. The segments such as odondoid, body, and pedicle and pars interarticularis are clear and it is easy to use in clinical practice.

\section{REFERENCES}

Anderson, L.D., D'Alonzo, R.T., 1974. Fractures of the odontoid process of the axis. J. Bone Joint Surg. 56, 16631674

Benzel, E.C., Hart, B.L., Ball, P.A., Baldwin, N.G., Orrison, W.W., Espinosa, M., 1994. Fractures of the C-2 vertebral body. J. Neurosurg. 81, 206-212

Benzel, E.C., 1996. Letter to the editor and response. Spine. 21, 2301-2302

Doherty, B.J., Heggeness, M.H., 1995. Quantitative anatomy of the second cervical vertebra. Spine. 20, 513-517

Doherty, B.J., Heggeness, M.H., Esses, S.I., 1993. A biomechanical study of odontoid fractures and fracture fixation. Spine. 18,178-184 